\title{
Fate of antibiotic resistance genes and its drivers during anaerobic co-digestion of food waste and sewage sludge based on microwave pretreatment
}

\author{
Junya Zhang ${ }^{1}$, Meixue Chen ${ }^{1}$, Qianwen Sui, Rui Wang, Juan Tong, Yuansong Wei *
}

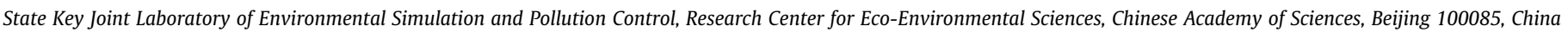
Department of Water Pollution Control Technology, Research Center for Eco-Environmental Sciences, Chinese Academy of Sciences, Beijing 100085, China

University of Chinese Academy of Sciences, Beijing 100049, China

\section{H I G H L I G H T S}

- AcoD reduced abundance of total ARGs compared with AD of mono-SS.

- AD effectively reduced both abundance and quantities of MRGs.

- AD of MW-SS was more effective than that of MW-FW for ARGs abundance control.

- Evolution of bacterial community was the main driver to the fate of ARGs.

- ARGs reduction may be associated with the decreased co-selection from heavy metals.

\section{A R T I C L E I N F O}

\section{Article history:}

Received 24 December 2015

Received in revised form 20 February 2016

Accepted 23 February 2016

Available online 8 March 2016

\section{Keywords:}

Antibiotic resistance genes

Sewage sludge

Food waste

Anaerobic digestion

Metal resistance genes

\begin{abstract}
A B S T R A C T
In this study, anaerobic digestion of mono-SS, MW-SS:FW and SS:MW-FW was investigated to understand the fate of ARGs and its drivers. Anaerobic digestion was effective for the reduction of metal resistance genes (MRGs), and could reduce the abundance of bla $a_{\mathrm{OXA}-1}$, sull and tet $G$, while sullI in co-digestion and $b a_{\mathrm{TEM}}$ and ereA only in MW-SS. ARGs reduction could be partly attributed to the reduction of co-selective pressure from heavy metals reflected by MRGs. However, the abundance of $m e f A / E$, ermB, ermF, tetM and tetX increased significantly. Anaerobic co-digestion, especially for MW-SS, could reduce total ARGs abundance compared with mono-SS, and evolution of bacterial community was the main driver for the fate of ARGs.
\end{abstract}

(c) 2016 Elsevier Ltd. All rights reserved.

\section{Introduction}

Increasing acquired antibiotic resistance is among the greatest worldwide concerns for health care, and it is currently considered to be one of the most serious public health issues. It has been estimated that antibiotic resistance is responsible for more than $25,000,23,000$, and 38,000 deaths every year in the European Union, the United States, and Thailand, respectively (Berglund, 2015). This highlights the need for broad strategies to slow the rate at which resistance spreads, and proactive treatment of anthro-

* Corresponding author at: State Key Joint Laboratory of Environmental Simulation and Pollution Control, Research Center for Eco-Environmental Sciences, Chinese Academy of Sciences, Beijing 100085, China. Tel./fax: +86 1062849690.

E-mail address: yswei@rcees.ac.cn (Y. Wei).

1 Junya Zhang and Meixue Chen contributed equally to the work. pogenic waste containing ARGs may help mitigate the spread of ARGs (Pruden et al., 2013).

Municipal wastewater treatment plants (WWTPs) were considered as significant reservoirs of ARGs, and numerous studies have detected amounts of ARGs at every stage of the municipal wastewater treatment processes (Munir et al., 2011). The vast majority of these ARGs are discharged from excess sludge, which has higher contribution (ca. 1000 times) to the release of the ARGs into the environment compared with effluent (Munir et al., 2011). In addition, ARGs were found in various foods like pork, beef, raw fruits, fresh vegetables, etc. (Rolain, 2013; Ruimy et al., 2010; Costa et al., 2008), while food waste (FW) in our daily life is composed of those things. Thus, FW should also be a reservoir of ARGs.

Ca. 6.25 million tons (dry solids) of sewage sludge (SS) were produced in 2013 at an average annual growth of 13\% from 2007 to 2013 in China (Yang et al., 2015), and ca. $6.0 \times 10^{7}$ tons of FW 
were produced according to China Statistical Yearbook 2011 with the annual increasing rate higher than $10 \%$ every year due to huge population and rising living standards (Zhang et al., 2016b). Anaerobic digestion (AD) due to the production of renewable energy was widely adopted to treat SS and FW, while low AD efficiency for SS caused by the slow hydrolysis process and $\mathrm{C} / \mathrm{N}$ ratio and the accumulation of volatile fatty acids (VFAs) for FW due to the labile organic fraction made the anaerobic co-digestion (AcoD) of SS and FW become increasingly popular, with the advantages of adjusting the $\mathrm{C} / \mathrm{N}$ ratio, increasing the methane yield, diluting harmful substances, and mediating the hydrolysis of FW and SS (Lee et al., 2009). Microwave pre-treatment (MW) has been proved to further enhance the AcoD (Zhang et al., 2016b). A few studies have investigated the fate of ARGs during AD of SS and suggested that $\mathrm{AD}$ could be used to reduce ARGs quantities (Ma et al., 2011; Diehl and Lapara, 2010; Ghosh et al., 2009). Generally, previous studies indicated that different ARGs responded differently under mesophilic or thermophilic conditions, with thermophilic digestion generally outperforming mesophilic digestion (Zhang et al., 2015). However, the fate of ARGs during the AcoD based on MW pretreatment has never been investigated. It may also contribute to the enhancement of ARGs reduction, and this deserves to be elucidated.

The co-occurrence of antibiotic and metal resistance in bacteria has been widely observed (Pal et al., 2015), which is caused by the cross- or co-resistance phenomena. Cross-resistance occurs when the same mechanism reduces the susceptibility to metals and antibiotics simultaneously, and co-resistance occurs when separate resistance genes are situated on the same genetic element (Pal et al., 2015). This fact may be of great importance in the case of SS, because it both contains significant amounts of heavy metals and antibiotics (Bondarczuk et al., 2016; Le-minh et al., 2010). Therefore, the presence of heavy metals in SS and its further treatment like land application may select for antibiotic-resistant bacteria, and it is imperative to figure out the evolution and effects of co-selection from heavy metals to ARGs during AD.

Thus, AD of mono-SS, MW-SS:FW (3:2, total solids, TS) and SS: MW-FW (3:2, TS) was carried out as previously suggested (Zhang et al., 2016b) to investigate the fate of eleven frequently detected ARGs in this study, as well as the evolution of class 1 integron (intI1), as the representative of mobile genetic elements (MGEs) and multiple resistance, and three plasmid-borne heavy metal resistance genes (MRGs, pcoA, $\operatorname{cop} A$ and $c z c A$ ) representing the co-selection of heavy metals was followed. The aims of this study were to (1) find out whether AcoD of SS and FW could contribute to reduction of ARGs and MRGs in SS and FW; (2) determine the main driver influencing the fate of ARGs regarding to bacterial community, MGEs and co-selection of heavy metals in order to provide some basic advice on ARGs control in SS and FW during AD.

\section{Methods}

\subsection{Experimental set-up}

Food waste (FW) was collected from the dining hall of Research Center for Eco-Environmental Sciences, Chinese Academy of Sciences, Beijing, China. After the removal of the hard matters and grease, FW was homogenized and crushed to particle size of ca. $2 \mathrm{~mm}$ and stored at $4{ }^{\circ} \mathrm{C}$ before use (TS, $136.83 \pm 11.65 \mathrm{~g} / \mathrm{L}$ ). The feed sludge (TS, $125.87 \pm 7.56 \mathrm{~g} / \mathrm{L}$ ) was the dewatered sewage sludge collected from Beijing Qinghe WWTP. Seeding sludge was collected from a mesophilic AD reactor treating SS in Beijing Xiaohongmen WWTP. The optimized ratio (3:2, total solids) of FW and SS for AcoD based on MW pretreatment was adopted. Briefly, SS and FW were mixed thoroughly at the optimized ratio (3:2) according to total solid (TS). $1.8 \mathrm{~L}$ of the mixture of substances and inoculums at a ratio of 5:1 (TS) were transferred to each bottle, and the final TS was adjusted to about $7 \%$. Then the bottles were incubated in a water bath to control temperature at $37 \pm 0.5^{\circ} \mathrm{C}$. Details of the optimization, biogas production, chemical parameters and the evolution of bacterial community were presented in a previous report (Zhang et al., 2016b).

\subsection{DNA extraction}

Samples on days $1,5,12,19$ and 33 at the optimized ratio of FW and SS based on MW were adopted for DNA extraction. $4 \mathrm{~mL}$ of each sludge sample was centrifuged at $10,000 \mathrm{rpm}$ for $10 \mathrm{~min}$, and the pellet was used for DNA extraction using FASTDNA Spin Kit for Soil (MP Biomedicals, USA) in triplicate according to manufacturer's instructions, and the resulting extracts were composited to average out bias in sampling and extraction. Quality and concentration of the extracted DNA were determined through 1\% agarose gel electrophoresis and NanoDrop ND-1000 (NanoDrop, USA), respectively.

\subsection{Quantitative $P C R(q P C R)$}

Eleven frequently detected ARGs including two $\beta$-lactam resistance genes (bla $a_{\mathrm{OXA}-1}$ and $b l a_{\mathrm{TEM}}$ ), four macrolide resistance genes ( $m e f A / E$, ereA, ermB and ermF), two sulfonamides resistance genes (sulI and sulII) and three tetracycline resistance genes (tetG, tetM and tetX) were quantified by qPCR. These ARGs were selected based on their frequent detection in previous studies (Ma et al., 2011; Diehl and Lapara, 2010; Zhang et al., 2016a) and their representative resistance mechanisms in the target antibiotics (Table 1). Meanwhile, evolution of three heavy metal resistance genes (MRGs, $p c o A, \operatorname{cop} A$ and $c z c A$ ), one representative mobile genetic element (class I integron, intI1) and 16s rRNA representing the biomass were determined. The primers used here and their corresponding target antibiotics and mechanisms were summarized in Table 1 . The plasmids containing these specific genes, used as standards in a 10-fold dilution for making qPCR standard curve, were manufactured by Zhejiang Tianke Biotechnology Company (Zhejiang, China). The $25 \mu \mathrm{L}$ PCR reaction mixtures contained $12.5 \mu \mathrm{L}$ of SYBR Green qPCR Super-Mix-UDG with Rox (Invitrogen, USA), $0.5 \mu \mathrm{L}$ each of $10 \mu \mathrm{M}$ forward and reverse primers (final concentration, $0.2 \mu \mathrm{M}$ ), $10.5 \mu \mathrm{L}$ of DNA-free water, and $10 \mathrm{ng}$ of standard plasmid or DNA extract. The thermo-cycling steps for qPCR amplification were as follows: (1) $50{ }^{\circ} \mathrm{C}, 2 \mathrm{~min}$; (2) $95^{\circ} \mathrm{C}, 5 \mathrm{~min}$; (3) $95{ }^{\circ} \mathrm{C}, 20 \mathrm{~s}$; (4) annealing temperature, $30 \mathrm{~s}$; (5) $72{ }^{\circ} \mathrm{C}, 31 \mathrm{~s}$; (6) plate read, repeat steps (3) through (5) 39 more times; (7) meltcurve analysis: $60-95^{\circ} \mathrm{C}, 0.2{ }^{\circ} \mathrm{C}$ read. The reaction was conducted using an ABI Real-time PCR system 7500 (ABI, USA). Primer specificity was confirmed by melting curves and gel electrophoresis. Each gene was quantified in triplicate for each sample using a standard curve and a negative control. The amplification efficiencies were between $90.3 \%$ and $100.1 \%$ and summarized in Table S1.

\subsection{Data analysis}

The generation of plots for the target genes was performed with OriginPro 9.0 (OriginLab, USA), and Excel 2013 (Microsoft, USA) was used to determine the averages and fold change values of ARGs. The gene copies indicated the absolute copy numbers present per unit of dry weight (DW), while the normalized copy number by $16 \mathrm{~S}$ rRNA was regarded as the abundance. The Spearman correlation was performed using SPSS 21.0 (IBM, USA), and a $p$ value $<0.05$ was considered statistically significant. Principal component analysis (PCA), redundancy analysis (RDA), partial RDA and 
Table 1

Primers used in this study and their corresponding target and mechanisms.

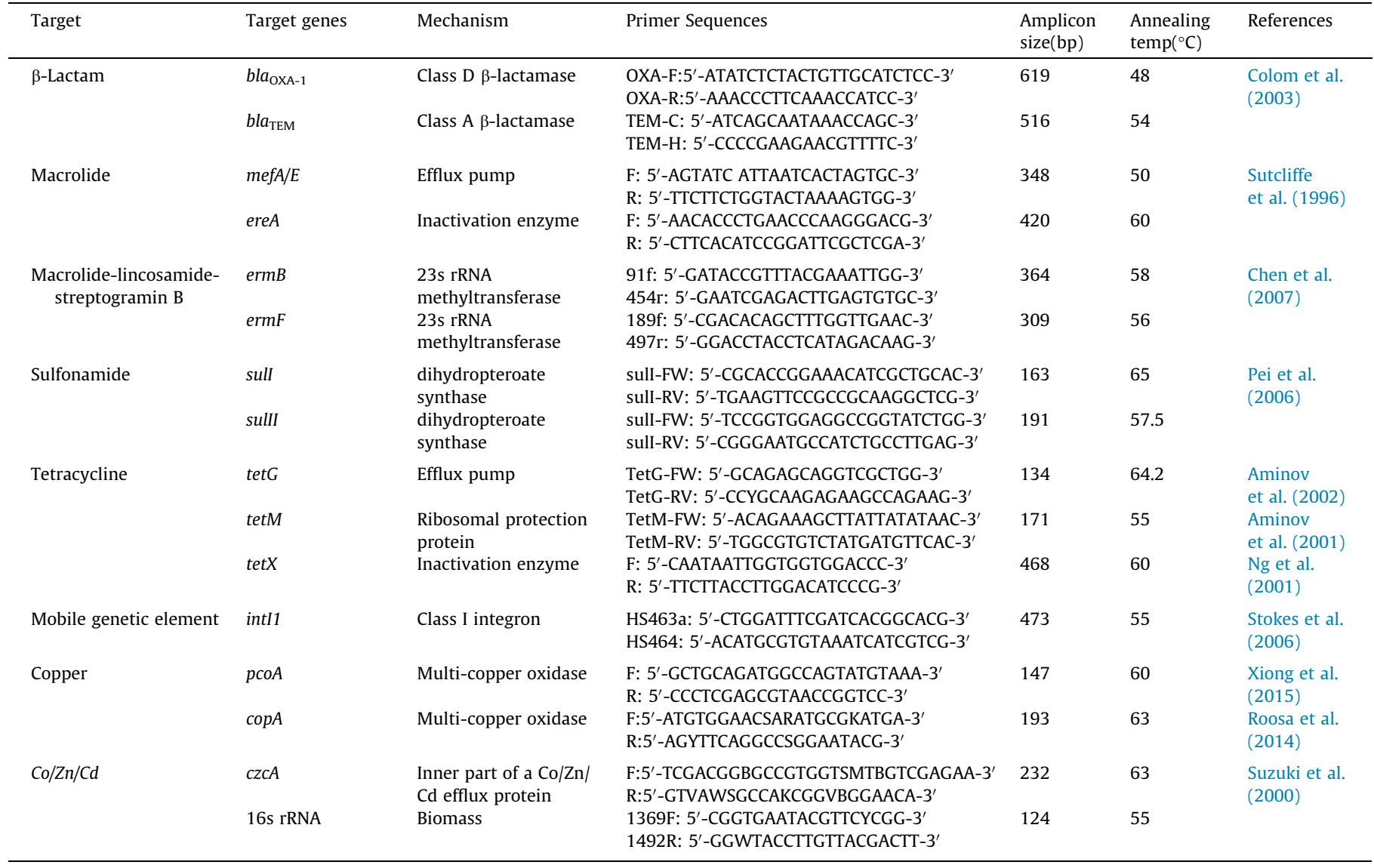

Procrustes analysis were conducted using Canoco 5.0 (Microcomputer Power, USA). Bacterial community has been analyzed previously using high-throughput sequencing method targeting the $16 \mathrm{~S}$ rRNA V4 region (Zhang et al., 2016b). To clarify the correlation between the evolution of ARGs and the bacterial community, a Mantel test was conducted using PAleontological STatistics software (PAST 3.07). The heatmap illustrating the evolution of the quantified genes in each sample was built by Heml 1.0. Network analysis based on the Spearman analysis between ARGs and the bacterial community (based on OTU), MRGs, intI1 and chemical parameters was determined using the Gephi platform (Gephi 0.8.2 beta).

\section{Results and discussion}

\subsection{Evolution of ARGs gene copies}

As shown in Fig. 1A and S1A, the ARGs in AcoD were significantly higher than mono-SS, which reflected that there were much more ARGs in FW than SS. The biomass reflected by $16 \mathrm{~S}$ rRNA increased significantly after $\mathrm{AD}$, and they all showed the same pattern that biomass increased on Day 5, and decreased on Day 12, further increased on Day 19 and then decreased. The absolute gene copies of total ARGs showed the similar pattern (Fig. S1), and total ARGs have significantly positive correlation $\left(p<10^{-4}\right)$ with biomass. This may be caused by the soluble organics released from the dewatered sludge after the hydrolysis during AD, and the biomass increased significantly at the presence of soluble organics which were reflected by the biogas production. However, not all ARGs gene copies had significantly positive correlation with biomass, e.g., bla $a_{\mathrm{OXA}-1}$, ereA, sulI and tetG. They showed different patterns responding to different treatments (Table 2). The gene copies of ereA and tetG showed some removal at the final for MW-SS, while increased in mono-SS and MW-FW, and sull decreased in MW-SS and MW-FW, while increased in mono-SS. Although bla $a_{\text {OXA-1 }}$ increased at the beginning, it decreased significantly at the final for all treatments, and even was not detected in the end of AD of the mono-SS and MW-SS.

As for the mono-SS in this study, the gene copies of all ARGs increased significantly except bla $a_{\mathrm{OXA}-1}$, although it also had the minimum biomass reflected by 16s rRNA in the feed (Fig. 1). In comparison with MW-FW, MW-SS showed some advantages in ARGs gene copies removal as reflected by Table 2. The sull dominated in the feed, but decreased in AcoD along with increasing in mono-SS. The gene copies of tetM, mefA/E, ermB and ermF increased significantly after $A D$, and became dominated in the final. Also, the gene copies of bla $a_{\mathrm{TEM}}$ increased after $\mathrm{AD}$. The significant increase of these genes after AD has been widely confirmed (Table 2). Interestingly, sulII and tetX increased significantly after AD in this study, while the controversial studies existed for the profiles of them, and not entire consistence of the ARG removal efficiency reported by these previous studies generally existed, e.g., Ma et al. (2011) indicated that mesophilic anaerobic digestion at both 10 and 20 day of solids retention times (SRTs) significantly reduced sulI, sulII, tetG, and tetX with longer SRT exhibiting a greater extent of removal, and Zhang et al. (2015) pointed out that sull increased significantly while tet $G$ decreased significantly at mesophilic AD through metagenomics approach. However, Yang et al. (2014) found that the abundance of tet $G$ changed little after AD. The reason for the inconsistency could be complicated, which might include the difference of digester operational parameters and feed sludge. Different from the primary and/or secondary sludge were 

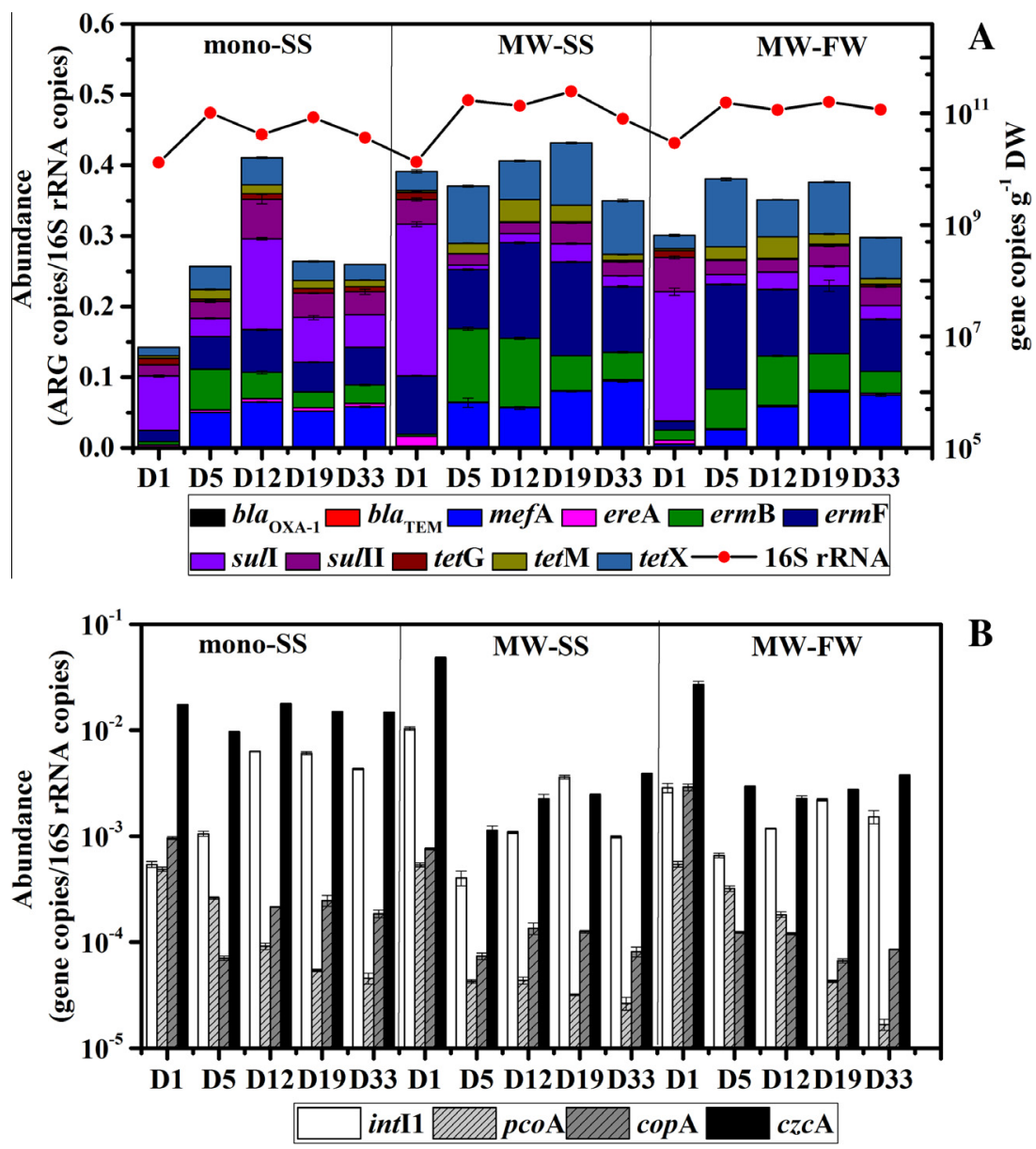

Fig. 1. Changes in the abundance of the ARGs (A), intI1 and heavy metals resistance genes (B) in different treatments during AD. D referred to day.

used previously in $\mathrm{AD}$, dewatered sludge was used as the feed in this study, and thus the initial abundance of ARGs may be quite different. For instance, the gene copies of sull could reach to ca. $10^{10} \mathrm{DW}^{-1}$ in previous study (Ma et al., 2011), while it was ca. $10^{9} \mathrm{DW}^{-1}$ in this study. Although the biomass reflected by $16 \mathrm{~s}$ rRNA was ca. $2.0 \times 10^{11} \mathrm{~g}^{-1} \mathrm{DW}$, it was ca. $1.31 \times 10^{10} \mathrm{~g}^{-1} \mathrm{DW}$ in this study, and the final biomass was almost the same (ca.1.0 $\times 10^{11} \mathrm{~g}^{-1} \mathrm{DW}$ ). Thus it was not comparable if the removal efficiency of ARGs was just considered as the comparison between the feed and the effluent.

\subsection{Fate of ARGs abundance}

MW-SS not only showed better biogas production than that of mono-SS and MW-FW as previously reported (Zhang et al., 2016b), but also were higher in the final reduction of the abundance of total ARGs (Fig. 1). The evolution of the absolute gene copies of ARGs indicated the profiles of the host bacteria during $A D$, and reflected the vertical gene transfer - normal gene replication due to the proliferation of the host bacteria. In order to determine the distribution of ARGs in response to the significant changes of the composition of bacterial community, each ARG was normalized to 16s rRNA. As shown in Fig. 2, the fate of each ARG was quite different in the same treatment and the fate of the same ARG was distinctive in different treatments. AcoD based on MW could reduce some ARGs abundance in comparison with mono-SS in which the abundance of ARGs increased significantly, and MW-SS showed better ARGs reduction than that of MW-FW (Fig. 1). This indicated that AcoD based on MW pre-treatment contributed to the ARGs reduction. The abundance of tet $G$ decreased in all treatments, and this was comparable to previous studies (Table 2), as well as the abundance of bla $a_{\mathrm{OXA}-1}$ (Fig. 2). Although the abundance of sull in all treatments decreased in this study the same as that by Ma et al. (2011), its increase was observed by Zhang et al. (2015) through metagenomics approach. The abundance of the three macrolide resistance genes (mefA/E, ermB and ermF) and tetM all increased (Fig. 2), and this was also comparable to previous studies (Table 2). The abundance of tetX increased in all treatments while decreased in previous studies. However, this may be not a bad thing, because the tet $X$ gene encodes for an NADPHrequiring oxidoreductase, which inactivates tetracycline in the presence of oxygen and NADPH, that is, it cannot function during $\mathrm{AD}$, but has only been found in a strict anaerobe, Bacteroides, where oxygen is excluded (Liu and Pop, 2009). The increase of the abundance of tetX after AD in this study could avail the degradation of tetracyclines for the further treatment at the presence of oxygen, and thus to reduce the selective pressure of tetracyclines. The abundance of sulII only decreased in AcoD but increased in mono-SS, while ereA and bla $a_{\mathrm{TEM}}$ only decreased in MW-SS. AcoD, especially for MW-SS, could reduce total ARGs abundance in comparison with mono-SS. The difference could be due to the different evolution of bacterial community.

\subsection{The role of intI1 and potential mechanisms of resistance}

An increase in gene abundance would suggest either that the organisms harboring genes encoding for resistance are multiplying or that the quantities of genes are increasing via horizontal gene 


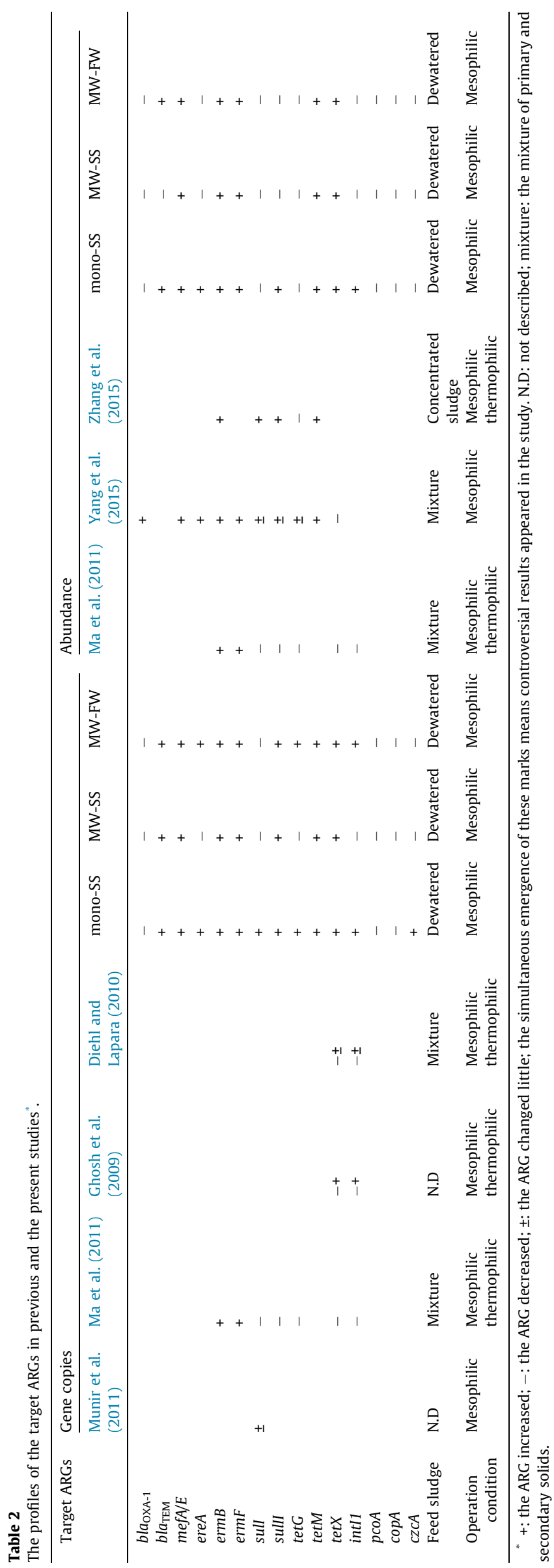

transfer (HGT). Mobile integrons like Class I integrons (intI1), were often used to represent the HGT. Although they cannot mobilize and transfer themselves between microbes, they are often associated with genetic elements which can, such as conjugative plasmids, transposons and insertion sequences (Berglund, 2015). Thus, intI1 was suggested as the proxy for anthropogenic pollution of ARGs (Gillings et al., 2014). Previous studies suggested that the abundance of intI1 could be reduced through AD with some exceptions (Table 2). In this study, intI1 could be reduced in AcoD, while increased in mono-SS, and intI1 had significantly positive correlation $(p<0.01)$ with ereA, sulI, sulli and tetG. This association was often observed previously, and might indicate that intI1 may be associated with their evolution.

The mechanisms of resistance to different antibiotics in $\mathrm{AD}$ were quite distinctive. The mechanism of tetracycline resistance during $\mathrm{AD}$ in this study may be the ribosomal protection protein reflected by the evolution of tetM, considering that tetX cannot function at the absence of oxygen and the decreasing abundance of tetG. As for macrolide resistance, it may be the efflux pump ( $m e f A / E)$ and 23s rRNA methyltransferase (ermB and ermF) that dominated the resistance. Class $A$ beta-lactamase, encoded by bla $a_{\mathrm{TEM}}$, exerted the resistance to beta-lactam. The TEM $\beta$ lactamase specified by plasmids in gram-negative bacteria are widely distributed in nature in terms of geographical distribution, association with plasmids of different incompatibility groups, and occurrence in different bacterial species, while the OXA- 1 are specified by plasmids of three, four, and two different incompatibility groups, respectively (Foster, 1983). Undoubtedly, the widespread potential host availed its dominance in AD. Furthermore, it is demonstrated that factors that can indirectly affect the level of $\beta$ lactamase expression and hence the resistance level of the cell are plasmid copy number, e.g., the TEM $\beta$-lactamase, which may be higher at anaerobic growth (Foster, 1983). While the pressure from sulfonamides may be reduced due to the effective degradation of sulfonamides during AD demonstrated by Mohring et al. (2009).

\subsection{Reduction of MRGs}

It has been concluded that copper, silver, arsenic, antimony, cobalt, nickel, cadmium, iron, zinc and mercury are all potential co-selectors for strains resistant to, e.g. sulfonamides, betalactams, amphenicols, tetracyclines and aminoglycosides, and certain bacterial taxa comprising many pathogens were particularly prone to carrying both MRGs and ARGs, highlighting the potential clinical consequences of co-selection (Pal et al., 2015). However, the bio-availability should be taken into account concerning the co-selection of heavy metals, because it is the bioavailable fractions of heavy metals that impose selective pressure on microbes. Moreover, MRGs could show better illustration of the real response of bacteria to the selective pressures caused by heavy metals (Roosa et al., 2014).

Unlike ARGs, all the MRGs quantified in this study decreased along with $\mathrm{AD}$, no matter considering the absolute gene copies (except $c z c A$ in mono-SS) or the abundance (Fig. 1B, 2 and S1B). The $\operatorname{cop} A$ is encoding for a periplasmic multi-copper oxidase which specified in plasmids, and this system needs oxygen contrary to the other systems such P-type ATPases and cation diffusion facilitators (Roosa et al., 2014). This could explain the reduction of copA abundance and gene copies in $\mathrm{AD}$ of this study. The pcoA (also a plasmid-borne $\mathrm{Cu}$ resistance) encodes a periplasmic multi-copper oxidase, and the pco gene cluster comprises seven genes pcoABCDRSE. Besides, the pco system requires CopA activity to confer resistance. Presumably, proteins encoded in the pco gene cluster are responsible for the handling of periplasmic copper delivered by CopA from the cytoplasm, and also there is significant 


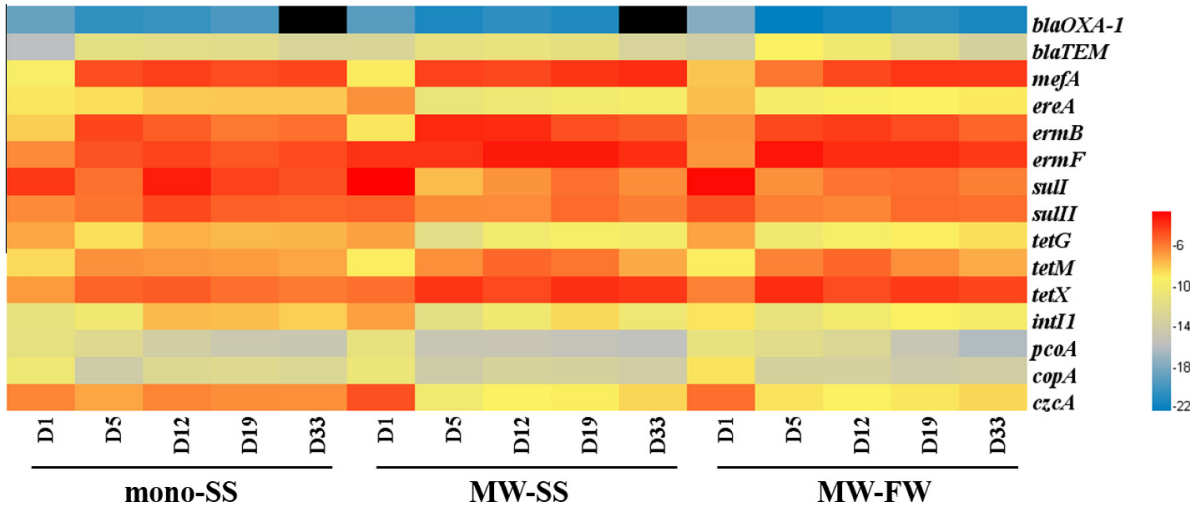

Fig. 2. Heatmap of changes in the target genes during AD in different treatments. Values plotted are the natural log2 transformed of the abundance of each ARG.

correlation between $p c o A$ and $\operatorname{cop} A$ in this study. The czc operon is driven by a $\mathrm{H}^{+}$ion gradient that allows $c z c A$ (plasmid-borne $\mathrm{Cd} / \mathrm{Zn} / \mathrm{Co}$ resistance) to pump heavy metals out of the cytoplasm, and it has been demonstrated that the measurement of $c z c A$ gene levels could be used to estimate $\mathrm{Cd} / \mathrm{Zn} / \mathrm{Co}$ bioavailability in sediment compartments (Roosa et al., 2014). This may indicate that the changes of the speciation of heavy metals happened, and bioavailability of heavy metals may be reduced during AD. Besides, there is no positively significant correlation $(p>0.05)$ between each MRG and 16s rRNA, and MRGs did not increased along with the increase of biomass. According to Spearman analysis, MRGs have some co-occurrence with specific ARGs. The ARGs decreased including bla $_{\mathrm{OXA}-1}$, ereA, sulI and tetG all have positively significant correlation $(p<0.05)$ with each MRG including $p c o A, \operatorname{cop} A$ and $c z c A$. Besides, the evolution of $c z c A$ was significantly correlated with sulII and intI1 which only decreased in AcoD. This indicated that the reduction of the selective pressure of heavy metals might favor the reduction of these ARGs.

\subsection{ARGs profile responding to the changes of bacterial community}

There was much difference of the evolution of bacterial community between mono-SS and MW-SS and MW-FW, and the details have been described as previously (Zhang et al., 2016b). The proliferation of ARGs still count on the activity of microbes, and the changes of bacterial community may have significant influence on the profiles of ARGs. In order to elucidate this, Procrustes analysis was conducted by rotating the ordination of changes in the bacterial community to match the profiles of ARGs based on PCA analysis, and the results indicated that there was positively significant correlation between the evolution of ARGs and bacterial community (Fig. 3). $77.2 \%$ of the variables of the evolution of ARGs could be explained by the changes of bacterial community through Procrustes analysis, and the correlation between the first two principal axes was significantly positive $(R=0.9734$ and 0.7787 , respectively). A further Mantel test based on Bray-Curtis distance confirmed this $(R=0.7833, p=0.0003$, permutation $N=9999)$. The evolution of bacterial community had significant correlation with the fate of ARGs.

A previous study confirmed that network analysis could be used to provide new insights into ARGs and their possible hosts in complex environmental scenarios (Zhang et al., 2016a). As shown in Fig. 4, various ARGs quantified in this study were significantly $(p<0.05)$ correlated with various species. The significantly positive correlation between different ARGs and the same host bacteria indicated by network analysis demonstrated the co-occurrence of different ARGs. The network was clearly divided into two groups: the ARGs that increased, and the ARGs that decreased (Fig. 4).

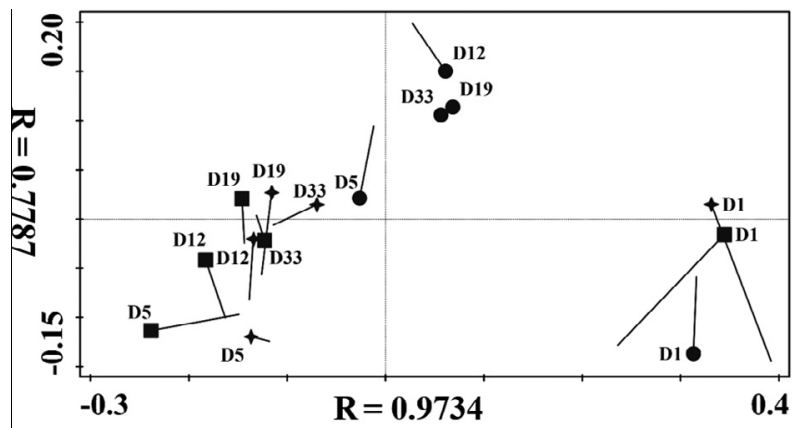

A

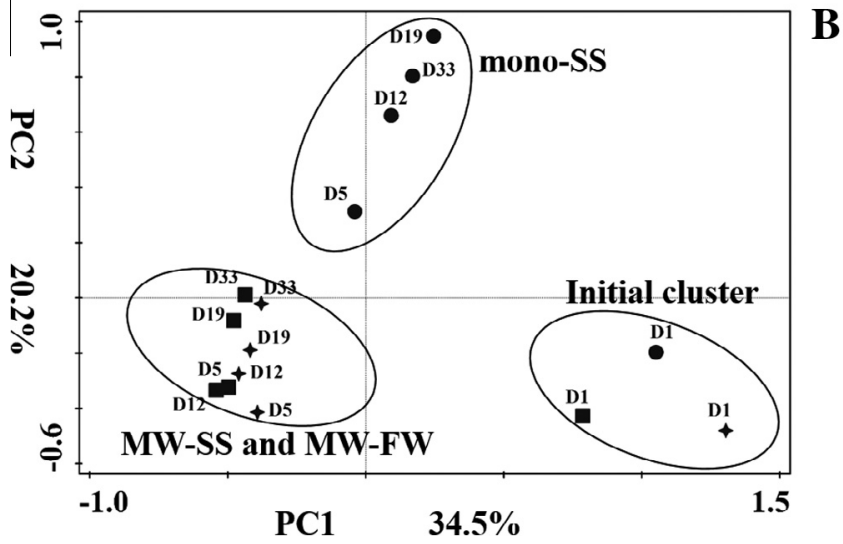

Fig. 3. Procrustes analysis of the significant correlation between ARG profiles and the bacterial community (A) and Principal component analysis (PCA) of the changes of the bacterial community for AD in different treatments (B). Circles, stars and triangles represent samples from mono-SS, MW-SS and MW-FW, respectively.

According to the network analysis, the genus Bacteroides may be the host bacteria of ermB, ermF, bla $a_{\mathrm{TEM}}$, tetM and tetX, while it has been widely demonstrated that ermB, ermF and tetX often existed in Bacteroides (Liu and Pop, 2009). The significant correlation between bla $_{\mathrm{TEM}}$, tetM and Bacteroides may be due to the functional correlation between their host bacteria and Bacteroides, or Bacteroides was their host bacteria through HGT, because tetM and $b l a_{\mathrm{TEM}}$ are often associated with conjugative transposons which also have a very wide host range (Liu and Pop, 2009). Their wide host range can also be reflected by the significant correlation with bacterial diversity index (Simpson) in Fig. 4. As for the ARGs decreased, the genus Aeromonas was the potential host bacteria of tet $G$, bla $a_{\mathrm{OXA}-1}$, ereA, sulI and sulII, and it has been demonstrated that Aeromonas was the host bacteria of ereA, sulI and sulII (Liu and Pop, 2009). Network analysis further elucidated the primary effects of bacterial community on the evolution of ARGs by 


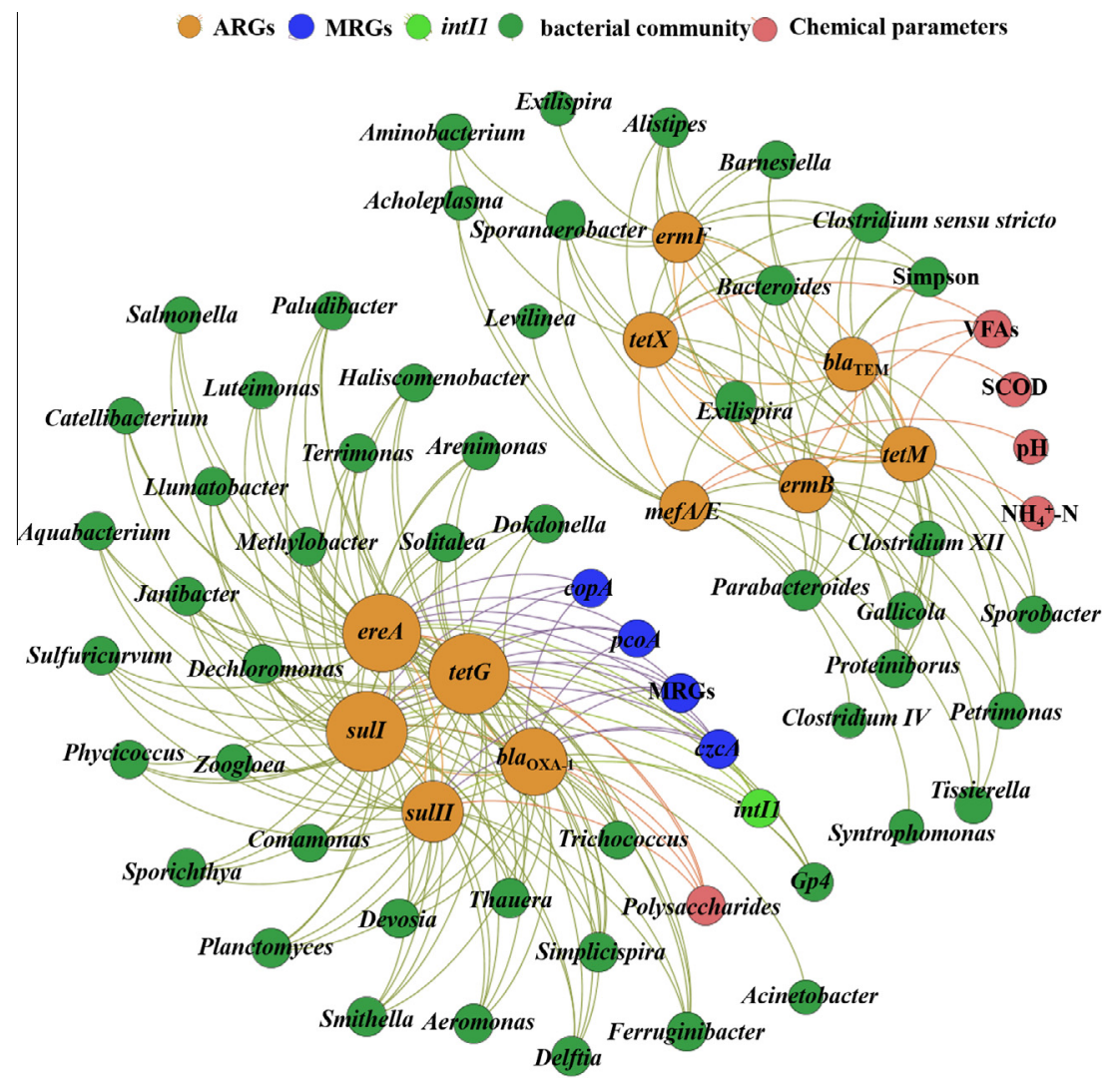

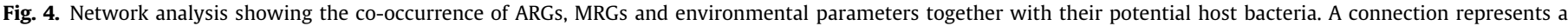
significant positive correlation $(p<0.05)$ according to spearman analysis.

determining their potential host bacteria. It has been demonstrated that environmental bacteria and pathogens harbor diverse and abundant ARGs (Forsberg et al., 2014). The potential host bacteria reflected by network analysis may be not antibiotic resistance bacteria (ARB) yet, and the significantly positive correlation with ARGs may be due to its functional connection with real ARBs. However, these potential host bacteria may have the biggest chance of becoming the ARBs through HGT due to their functional connection. This would make sense in case that the function connection was found between pathogens and non-pathogens.

3.6. The relationship among environmental factors, bacterial communities, MRGs and ARGs

RDA analysis was conducted to investigate the relationships between environmental factors, bacterial community, intI1 and MRGs and ARGs (Fig. 5), and the results showed that the selected variables accounted for $98.2 \%$ of the total variation of the evolution of ARGs quantified in this study $(p=0.002)$. The contributors for the ARGs profiles in different stages are quite different. Proteobacteria, Actinobacteria and Planctomycetes accounted for the patterns at the beginning, and could explain the evolution of the ARGs decreased including bla $a_{\mathrm{OXA}-1}$, sulI, sulII, tetG and ereA. Firmicutes mainly accounted for the evolution of the ARGs increased including tetX, bla $a_{\mathrm{TEM}}, e r m B$, ermF and tetM, while Bacteroides may account for the pattern of ARGs at D5 in MW-SS and MW-FW, and Firmicutes was also previously demonstrated to contribute to the elevated abundance and enrichment of ARGs (Zhang et al., 2016a). Thus, ARGs reduction by reducing the abundance of Firmicutes in $A D$ may be feasible, but the dominance of Firmicutes often occurred in various AD (Zhang et al., 2016b).

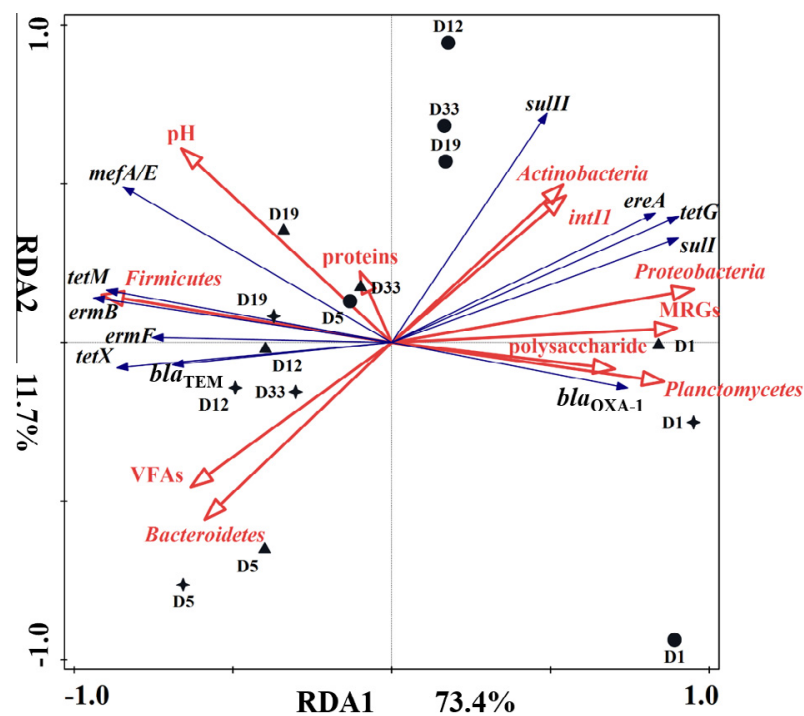

Fig. 5. Redundancy analysis (RDA) results of the relationship between environmental factors, bacterial community, MRGs and intI1 and ARGs profiles in AD of mono-SS, MW-SS and MW-FW, respectively. The blue lines and red lines represent the species variables and environmental variables, respectively, during the RDA analysis through Canoco 5.0 in this study. Black circle, star and triangle represented samples from mono-SS, MW-SS and MW-FW, respectively. (For interpretation of the references to colour in this figure legend, the reader is referred to the web version of this article.)

As far as the environmental factors were concerned in this study, there was significant correlation between $\mathrm{pH}$ and mefA/E (Fig. 4), and proteins correlated significantly to the patterns of 
ARGs at D2 for mono-SS, while polysaccharide was significantly correlated with the ARGs decreased. This indicated that changes of the environmental factor influenced the evolution of ARGs. For instance, the easier degradation of polysaccharide than proteins may lead to the dominance of fast growing host bacteria, and higher $\mathrm{pH}$ may resulted in the SOS response for some microbes, while the SOS could avail the increase of specific ARGs (Miller et al., 2014). To determine the key contributor to the explanatory variation as a whole and separate the influences of bacterial community, environmental factors concerned, intI1 and MRGs, partial RDA was conducted by designating the explanatory variables and covariates (Table S2). It was concluded that the bacterial community contributed the most to the ARG changes, followed by environmental factors, intI1 and MRGs. Indeed, the evolution of the bacterial community was the main driver for the changes in ARGs rather than HGT induced by MGEs or co-selection/co-occurrence determined by MRGs. The dominant contribution of the bacterial community to ARG profiles has been elucidated in various environments (Zhang et al., 2016a), including soils, rivers, WWTPs, sludge composting etc., and this was the first report that elucidated the dominant contribution of bacterial community in AD. Nevertheless, the less frequent HGT incidents should not be overlooked, as even one HGT event into a human pathogen has the potential for great harm (Zhang et al., 2016a).

Besides, the sludge retention time (SRT) was considered as one of the most important operation parameters for AD. It seemed the same importance for the ARGs reduction. The extension of SRT could favor the reduction of ARGs during AD, and the detail evolution of the abundance of each ARG was shown in Fig. 2 and Table S3. The abundance of ARGs increased to the maximum on D12 for both mono-SS and MW-FW, while it increased to the maximum on D19. But the best ARGs reduction occurred on D33. This may indicate that longer time was needed to exhibit a great extent of reduction ARGs during $\mathrm{AD}$, which was also demonstrated previously (Ma et al., 2011). From the point view of the abundance reduction of ARGs in this study, it seemed that the mono-SS showed the highest risks on Day12, while it was on Day19 for AcoD. The evolution of absolute gene copies of ARGs showed the same pattern (Fig. S1).

\section{Conclusions}

Anaerobic co-digestion (AcoD) showed some advantages over mono-SS digestion, and MW-SS was better than that of MW-FW considering both the methane production and the reduction of ARGs abundance. AcoD could reduce total ARGs abundance and should be adopted more widely concerning the ARGs control in SS and FW. The decrease of selective pressure from heavy metals due to AD may partially contribute to the reduction of ARGs. Evolution of bacterial community composition is the main driver for the fate of ARGs, not HGT.

\section{Acknowledgements}

This work is supported by the Special Fund for Agro-scientific Research in the Public Interest of China (No. 201303091), Major Science and Technology Program for Water Pollution Control and Treatment of China (2012ZX07202-005, 2015ZX07203-007) and the National Natural Science Foundation of China (21377151).

\section{Appendix A. Supplementary data}

Supplementary data associated with this article can be found, in the online version, at http://dx.doi.org/10.1016/j.biortech.2016.02. 140.

\section{References}

Aminov, R., Chee-Sanford, J., Garrigues, N., Teferedegne, B., Krapac, I., White, B., Mackie, R.I., 2002. Development, validation, and application of pcr primers for detection of tetracycline efflux genes of gram-negative bacteria. Appl. Environ. Microbiol. 68, 1786-1793.

Aminov, R.I., Garrigues-Jeanjean, N., Mackie, R.I., 2001. Molecular ecology of tetracycline resistance: development and validation of primers for detection of tetracycline resistance genes encoding ribosomal protection proteins. Appl. Environ. Microbiol. 67, 22-23.

Berglund, B., 2015. Environmental dissemination of antibiotic resistance genes and correlation to anthropogenic contamination with antibiotics. Infect. Ecol. Epidemiol. 5, 28564.

Bondarczuk, K., Markowicz, A., Piotrowska-Seget, Z., 2016. The urgent need for risk assessment on the antibiotic resistance spread via sewage sludge land application. Environ. Int. 87, 49-55.

Chen, J., Yu, Z., Michel, F.C., Wittum, T., Morrison, M., Michel, J., Frederick, C., Wittum, T., Morrison, M., 2007. Development and application of real-time PCR assays for quantification of erm genes conferring resistance to macrolideslincosamides-streptogramin B in livestock manure and manure management systems. Appl. Environ. Microbiol. 73, 4407-4416.

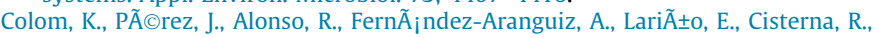
2003. Simple and reliable multiplex PCR assay for detection of blaTEM, blaSHV and blaOXA-1 genes in Enterobacteriaceae. FEMS Microbiol. Lett. 223, 147-151.

Costa, D., Poeta, P., Sáenz,, Y., Coelho, A.C., Matos, M., Vinue, L., Rodrigues, J., Torres, C., 2008. Prevalence of antimicrobial resistance and resistance genes in faecal Escherichia coli isolates recovered from healthy pets. Vet. Microbiol. 127, 97105

Diehl, D.L., Lapara, T.M., 2010. Effect of temperature on the fate of genes encoding tetracycline resistance and the integrase of class 1 integrons within anaerobic and aerobic digesters treating municipal wastewater solids. Environ. Sci. Technol. 44, 9128-9133.

Forsberg, K.J., Patel, S., Gibson, M.K., Lauber, C.L., Knight, R., Fierer, N., Dantas, G., 2014. Bacterial phylogeny structures soil resistomes across habitats. Nature 509, 612-616.

Foster, T.J., 1983. Plasmid-determined resistance to antimicrobial drugs and toxic metal ions in bacteria. Microbiol. Mol. Biol. Rev. 47, 361-409.

Ghosh, S., Ramsden, S.J., LaPara, T.M., 2009. The role of anaerobic digestion in controlling the release of tetracycline resistance genes and class 1 integrons from municipal wastewater treatment plants. Appl. Microbiol. Biotechnol. 84 $791-796$.

Gillings, M.R., Gaze, W.H., Pruden, A., Smalla, K., Tiedje, J.M., Zhu, Y.-G., 2014. Using the class 1 integron-integrase gene as a proxy for anthropogenic pollution. ISME J. 9, 1269-1279.

Lee, M., Hidaka, T., Hagiwara, W., Tsuno, H., 2009. Comparative performance and microbial diversity of hyperthermophilic and thermophilic co-digestion of kitchen garbage and excess sludge. Bioresour. Technol. 100, 578-585.

Le-minh, N., Khan, S.J., Drewes, J.E., Stuetz, R.M., 2010. Fate of antibiotics during municipal water recycling treatment processes. Water Res. 44, 4295-4323.

Liu, B., Pop, M., 2009. ARDB-antibiotic resistance genes database. Nucleic Acids Res. 37, 443-447.

Ma, Y., Wilson, C.a., Novak, J.T., Riffat, R., Aynur, S., Murthy, S., Pruden, A., 2011. Effect of various sludge digestion conditions on sulfonamide, macrolide, and tetracycline resistance genes and class I integrons. Environ. Sci. Technol. 45, $7855-7861$.

Miller, J.H., Novak, J.T., Knocke, W.R., Pruden, A., 2014. Elevation of antibiotic resistance genes at cold temperatures: implications for winter storage of sludge and biosolids. Lett. Appl. Microbiol. 59, 587-593.

Mohring, S.a.I., Strzysch, I., Fernandes, M.R., Kiffmeyer, T.K., Tuerk, J., Hamscher, G., 2009. Degradation and elimination of various sulfonamides during anaerobic fermentation: a promising step on the way to sustainable pharmacy. Environ. Sci. Technol. 43, 2569-2574

Munir, M., Wong, K., Xagoraraki, I., 2011. Release of antibiotic resistant bacteria and genes in the effluent and biosolids of five wastewater utilities in Michigan. Water Res. 45, 681-693.

Ng, L.K., Martin, I., Alfa, M., Mulvey, M., 2001. Multiplex PCR for the detection of tetracycline resistant genes. Mol. Cell. Probes 15, 209-215.

Pal, C., Bengtsson-palme, J., Kristiansson, E., Larsson, D.G.J., 2015. Co-occurrence of resistance genes to antibiotics, biocides and metals reveals novel insights into their co-selection potential. BMC Genomics 16, 964.

Pei, R., Kim, S.-C., Carlson, K.H., Pruden, A., 2006. Effect of river landscape on the sediment concentrations of antibiotics and corresponding antibiotic resistance genes (ARG). Water Res. 40, 2427-2435.

Pruden, A., Joakim Larsson, D.G., Amézquita, A., Collignon, P., Brandt, K.K., Graham, D.W., Lazorchak, J.M., Suzuki, S., Silley, P., Snape, J.R., Topp, E., Zhang, T., Zhu, Y. G., 2013. Management options for reducing the release of antibiotics and antibiotic resistance genes to the environment. Environ. Health Perspect. 121, $878-885$.

Rolain, J.M., 2013. Food and human gut as reservoirs of transferable antibiotic resistance encoding genes. Front. Microbiol. 4, 1-10.

Roosa, S., Wattiez, R., Prygiel, E., Lesven, L., Billon, G., Gillan, D.C., 2014. Bacterial metal resistance genes and metal bioavailability in contaminated sediments. Environ. Pollut. 189, 143-151.

Ruimy, R., Brisabois, A., Bernede, C., Skurnik, D., Barnat, S., Arlet, G., Momcilovic, S., Elbaz, S., Moury, F., Vibet, M.A., Courvalin, P., Guillemot, D., Andremont, A., 
2010. Organic and conventional fruits and vegetables contain equivalent counts of Gram-negative bacteria expressing resistance to antibacterial agents. Environ. Microbiol. 12, 608-615.

Stokes, H.W., Nesbø, C.L., Holley, M., Bahl, M.I., Gillings, M.R., Boucher, Y., 2006. Class 1 integrons potentially predating the association with Tn402-like transposition genes are present in a sediment microbial community. J. Bacteriol. 188, 5722-5730.

Sutcliffe, J., Grebe, T., Wondrack, L., Sutcliffe, J., Grebe, T., Tait-Kamradt, A. Wondrack, L., 1996. Detection of erythromycin-resistant determinants by PCR. Antimicrob. Agents Chemother. 40, 2562-2566.

Suzuki, M.T., Taylor, L.T., Delong, E.F., Long, E.F.D.E., 2000. Quantitative analysis of small-subunit rrna genes in mixed microbial populations via $5^{\prime}$-nuclease assays quantitative analysis of small-subunit rRNA genes in mixed microbial populations via 5J-nuclease assays. Appl. Environ. Microbiol. 66, 4605-4614.

Xiong, W., Zeng, Z., Zhang, Y., Ding, X., Sun, Y., 2015. Fate of metal resistance genes in arable soil after manure application in a microcosm study. Ecotoxicol. Environ. Saf. 113, 59-63.
Yang, G., Zhang, G., Wang, H., 2015. Current state of sludge production, management, treatment and disposal in China. Water Res. 78, 60-73.

Yang, Y., Li, B., Zou, S., Fang, H.H.P.P., Zhang, T., 2014. Fate of antibiotic resistance genes in sewage treatment plant revealed by metagenomic approach. Water Res. 62, 97-106.

Zhang, J., Chen, M., Sui, Q., Tong, J., Jiang, C., Lu, X., Zhang, Y., Wei, Y., 2016a. Impacts of addition of natural zeolite or a nitrification inhibitor on antibiotic resistance genes during sludge composting. Water Res. 91, 339-349.

Zhang, J., Lv, C., Tong, J., Liu, J., Liu, J., Yu, D., Wang, Y., Chen, M., Wei, Y., 2016b. Optimization and microbial community analysis of anaerobic co-digestion of food waste and sewage sludge based on microwave pretreatment. Bioresour. Technol. 200, 253-261.

Zhang, T., Yang, Y., Pruden, A., 2015. Effect of temperature on removal of antibiotic resistance genes by anaerobic digestion of activated sludge revealed by metagenomic approach. Appl. Microbiol. Biotechnol. 99, 7771-7779. 